\title{
Shock emission in the bipolar post-AGB star IRAS 16594-4656
}

\author{
G. C. Van de Steene ${ }^{1}$ and P. A. M. van Hoof ${ }^{2,3}$ \\ 1 Royal Observatory of Belgium, Ringlaan 3, 1180 Brussels, Belgium \\ 2 APS Division, Physics Dept., Queen's University of Belfast, BT7 1NN, Northern Ireland \\ 3 Canadian Institute for Theoretical Astrophysics, McLennan Labs, University of Toronto, 60 St. George St., \\ Toronto, ON M5S 3H8, Canada
}

Received 11 December 2002 / Accepted 12 May 2003

\begin{abstract}
In this paper we study the near-infrared emission spectrum of IRAS 16594-4656, a bipolar post-AGB star with spectral type B7 and no observed ionization. Using optical and near-infrared photometry we determined the total extinction towards this object to be $A_{V}=7.5 \pm 0.4 \mathrm{mag}$ and derived a distance of $2.2 \pm 0.4 \mathrm{kpc}$, assuming a luminosity of $10^{4} L_{\odot}$. The near-infrared spectrum shows strong $\mathrm{H}_{2}$ emission lines and some typical metastable shock excited lines such as [Fe II] $1.257 \& 1.644 \mu \mathrm{m}$. We determined the rotational and vibrational excitation temperatures, as well as the ortho-to-para ratio of the molecular hydrogen. Based on these we argue that the $\mathrm{H}_{2}$ emission is mainly collisionally excited. Line ratios indicate that the $\mathrm{H}_{2}$ emission originates in a $\sim 25 \mathrm{~km} \mathrm{~s}^{-1} C$-type shock. On the other hand, the metastable lines, and especially the [Fe II] emission lines, indicate the presence of a $\sim 75 \mathrm{~km} \mathrm{~s}^{-1} J$-type shock. Hence we postulate that the $\mathrm{H}_{2}$ emission originates where the stellar wind (with an observed terminal velocity of $\sim 126 \mathrm{~km} \mathrm{~s}^{-1}$ ) is funneled through an equatorial density enhancement, impinging almost tangentially upon the circumstellar material. The [Fe II] emission either occurs along the walls of the bipolar lobes where the transverse shock velocity would be higher, or could originate much closer to the central star in shocks in the post-AGB wind itself, or possibly even an accretion disk. Further high resolution near-infrared spectra are currently being obtained to confirm the proposed geometry and kinematics.
\end{abstract}

Key words. hydrodynamics - shock waves - stars: AGB and post-AGB - stars: winds, outflows - ISM: molecules infrared: ISM

\section{Introduction}

Post-AGB stars represent an important transition phase in the evolution of low and intermediate-mass stars, between the asymptotic giant branch (AGB) and the planetary nebula (PN) phases. During this period, the detached circumstellar envelope of gas and dust is expanding away from the star. Meanwhile the star itself is increasing in temperature at about constant luminosity. This phase lasts a few thousand years depending upon the star's core mass. When the temperature is high enough and the star photo-ionizes the nebula, it has entered the PN phase.

In spite of extensive study, the evolution from the AGB toward the PN stage is still poorly understood. The drastic changes observed in circumstellar structure and kinematics are particularly puzzling. During late AGB or early post-AGB evolutionary stages, the geometry of the circumstellar material changes from more or less spherically symmetric to axially symmetric, with the result that most PNe exhibit axisymmetric structures, ranging from elliptical to bipolar. Bipolar PNe

Send offprint requests to: G. C. Van de Steene,

e-mail: gsteene@oma.be

* Based on observations collected at the European Southern Observatory, Chile (proposal No. 61.C-0567). are very likely to possess molecular envelopes that are readily detectable in the near-infrared ro-vibrational lines of $\mathrm{H}_{2}$ (Kastner et al. 1996). The available data suggest that the onset of near-infrared $\mathrm{H}_{2}$ emission in PNe can be traced back to the pre-planetary nebula (PPN) phase but not back to the AGB phase of evolution (Weintraub et al. 1998). These observations suggest that further studies of $\mathrm{H}_{2}$ emission from PPNe may offer insight into the transition from AGB star to PN and from spherical to axisymmetric mass loss.

The study of transition objects showing $\mathrm{H}_{2}$ emission at an early stage is crucial for understanding the hydrodynamic processes shaping the nebulae. The $\mathrm{H}_{2}$ lines can reveal details about the physical conditions in the shocks associated with these hydrodynamic processes and thereby help constrain models of the interaction of the central star with the AGB remnant. Only 13 post-AGB stars with $\mathrm{H}_{2}$ emission have been detected in molecular hydrogen (García-Hernández et al. 2002). RAFGL 2688 (the Egg Nebula) and RAFGL 618 are perhaps the best-studied examples of such transition objects and the only two whose $\mathrm{H}_{2}$ emission has been extensively studied. Both objects display molecule-rich circumstellar envelopes, carbon-rich circumstellar chemistries, and dusty, bipolar reflection nebulosities. Whereas RAFGL 2688 harbors an 
F-type central star and is not known to contain an $\mathrm{H}$ II region, RAFGL 618 possesses a B-type central star and does contain a compact HII region, suggesting that the latter nebula has evolved further toward the PN stage. In this paper we study in detail the excitation mechanism of the $\mathrm{H}_{2}$ emission in the PPN IRAS 16594-4656.

IRAS 16594-4656 is classified as a post-AGB star for several reasons. In the IRAS color-color diagram it has the colors of a planetary nebula (PN) (Van de Steene \& Pottasch 1993), but it has never been detected in the radio (Van de Steene et al. 2000a). It has a large infrared excess due to dust with a color temperature of $173 \mathrm{~K}$. It displays a double-peaked spectral energy distribution, with the peak in the mid-infrared much brighter than the peak in the near-infrared (Van de Steene et al. 2000a). It possesses a CO envelope with an expansion velocity of at least $16 \mathrm{~km} \mathrm{~s}^{-1}$ (Loup et al. 1990). The chemistry of IRAS 16594-4656 appears to be carbon-rich. This is based on the detection of unidentified IR emission features at 3.3, 6.2, $7.7,8.6,11.3,12.6$, and $13.4 \mu \mathrm{m}$, as well as the $21 \mu \mathrm{m}$ feature (García-Lario et al. 1999), all commonly associated with a carbon-rich chemistry. However, tentative detections of crystalline silicate features (mostly pyroxenes) were also reported by García-Lario et al. (1999). The latter are associated with an oxygen-rich chemistry, which could point to the fact that IRAS 16594-4656 only turned carbon-rich during one of the most recent thermal pulses.

The optical spectrum of IRAS 16594-4656 shows a spectral type B7 with significant reddening. The Balmer lines of hydrogen are in emission, together with a weak [O I] emission at $6300 \AA$. The $\mathrm{H} \alpha$ emission has a P-Cygni type profile indicative of a stellar wind with a terminal velocity of approximately $126 \mathrm{~km} \mathrm{~s}^{-1}$ (Van de Steene et al. 2000b).

In the optical the nebulosity is dominated by scattered light, not emission lines as in the case of planetary nebulae (Hrivnak et al. 1999). The HST image of IRAS 16594-4656 shows a bipolar morphology (Hrivnak et al. 1999), with the southwestern lobe tilted towards us at an intermediate orientation ( $\mathrm{Su}$ et al. 2001), as well as concentric arcs (Hrivnak et al. 2001). $\mathrm{Su}$ et al. (2000) noted that in addition to the centro-symmetric polarimetric patterns, point-symmetric patterns are also seen in IRAS 16594-4656. Such patterns provide strong, independent evidence for the presence of a circumstellar disk or torus (hereafter referred to as an equatorial density enhancement or EDE).

Spectroscopic studies of $\mathrm{H}_{2}$ are particularly useful because the intensity ratios of $\mathrm{H}_{2}$ lines arising from different excited levels provide diagnostics of the excitation mechanism and physical conditions. There are two likely excitation mechanisms for $\mathrm{H}_{2}$ in post-AGB stars: UV pumping ("fluorescence") by stellar photons, and thermal (i.e., collisional) excitation by the gas. It is possible that both mechanisms play a role within a single post-AGB star. All spectroscopic (and imaging) studies of post-AGB stars to date have concentrated only on the strong $\mathrm{H}_{2}(1,0) \mathrm{S}(1) 2.121 \mu \mathrm{m}$ and $\mathrm{H}_{2}(2,1) \mathrm{S}(1) 2.247 \mu \mathrm{m}$ spectral lines. Their ratio can in principle distinguish between the two excitation mechanisms. However, its utility as a diagnostic breaks down at high densities, when collisions modify the populations of the lowest vibrational levels $(v=1,2)$, driving them to their thermal values, even when the gas is

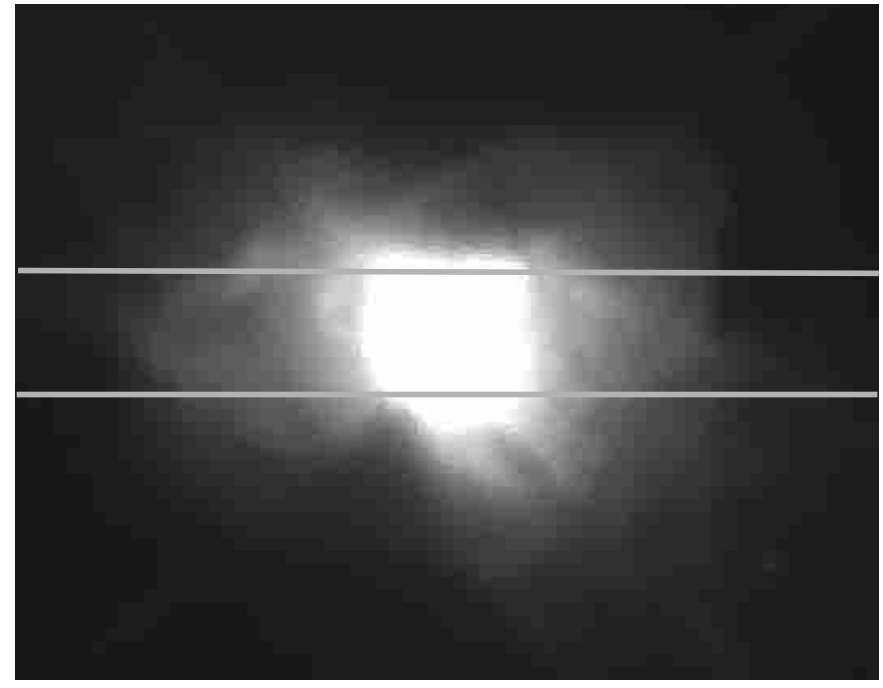

Fig. 1. The HST mage of IRAS 16594-4656 through filter F814W (Hrivnak et al. 1999) with the slit position indicated by the grey lines. North is to the top and East to the left.

fluorescently excited. Emission lines from higher vibrational levels are less affected, and can still distinguish gas in which UV photons are responsible for the $\mathrm{H}_{2}$ excitation. Furthermore time-dependent effects might enhance the intensity of UVpumped emission soon after the onset of far-UV irradiation, enabling fluorescent $\mathrm{H}_{2}$ emission to rival the high surface brightness typical of shocked gas. This makes it particularly important to study more than a handful of post-AGB stars, over a large spectral range, in order to achieve a broad, balanced picture of their molecular components.

For the first time we present a full $J$-, $H$-, $K$-band spectrum of a post-AGB star. The observations are presented in Sect. 2. The extinction and distance are derived in Sect. 3 and the infrared spectrum is discussed in Sect. 4. Many $\mathrm{H}_{2}$ emission lines were detected and we consider them all to discuss the excitation mechanism in Sect. 5. The results are discussed in Sect. 6 and final conclusions are given in Sect. 7.

\section{Observations}

The near-infrared spectrum of IRAS 16594-4656 was obtained with SOFI on the NTT telescope at La Silla (ESO) in June 1998. The slit width was $1^{\prime \prime}$ and the slit orientation almost eastwest. An image with the slit position indicated is presented in Fig. 1. The SOFI pixel scale is 0.'29 perpixel. Between 0.95 and $1.65 \mu \mathrm{m}$ the spectrum was obtained with the GB grism and has a spectral resolution of $7 \AA$ per pixel. Between 1.53 and $2.52 \mu \mathrm{m}$ the spectrum was obtained with the GR grism and has a resolution of $10.2 \AA$ per pixel. The seeing was 0 '. $^{\prime} 7$. The red spectrum is an average of 6 cycles of 10 DITs of $1.5 \mathrm{~s}$ and the blue spectrum an average of 6 cycles of 10 DITs of $2 \mathrm{~s}$. The airmass was 1.45 .

The spectra were reduced with an adapted version of the ESO package ECLIPSE and further processed and analyzed in IRAF. A selection of 3 standard stars, observed throughout the night, was used to construct the response curve. To obtain absolute flux tables for the standard stars, we constructed theoretical 
Table 1. The observed and intrinsic magnitudes of IRAS 16594-4656. The intrinsic magnitudes are arbitrarily scaled to $V=0 \mathrm{mag}$.

\begin{tabular}{lrrrrrrr}
\hline \hline & $B^{a}$ & $V^{a}$ & $R_{\mathrm{C}}^{a}$ & $I_{\mathrm{C}}^{a}$ & $J^{b}$ & $H^{b}$ & $K^{b}$ \\
& mag & mag & mag & mag & mag & mag & mag \\
\hline$m_{\text {obs }}$ & 16.31 & 14.60 & 13.27 & 12.00 & 9.73 & 8.85 & 8.20 \\
$m_{\text {intr }}$ & -0.09 & 0.00 & 0.01 & 0.06 & 0.16 & 0.18 & 0.23 \\
\hline
\end{tabular}

${ }^{a}$ Hrivnak et al. (1999).

${ }^{b}$ Van de Steene et al. (2000a).

spectra for the standard stars based on NextGen atmosphere models produced with the stellar atmosphere code PHOENIX (Hauschildt et al. 1999), which were subsequently convolved to the SOFI resolution and wavelength grid. Next we included an extinction component and scaled the spectra to match the observed $J_{-}, H$-, and $K$-band flux values. These spectra were compared with the observed ones to derive the average response curve. The object spectra were calibrated with this smoothed response curve. Comparison of the observed, calibrated spectra of the standard stars and the theoretical spectra provided us with the spectrum of the atmosphere, which was subsequently used to correct for telluric absorption.

\section{Extinction and distance}

IRAS $16594-4656$ lies very close to the Galactic plane ( $b=$ $-3.3)$. As such, it is expected to be significantly reddened. Because the reddening must be accounted for in the analysis of the emission lines and the continuum, we begin this section with a determination of the reddening. We used $B V R_{\mathrm{C}} I_{\mathrm{C}}$ photometry taken from Hrivnak et al. (1999) and JHK photometry taken from Van de Steene et al. (2000a). We excluded the observed $L$-band magnitude from our analysis since it is apparently contaminated by emission from either atomic lines or hot dust. The spectral type of the central star is B7 (Van de Steene et al. 2000b) and we assumed that the star has a low surface gravity. The intrinsic colors of the central star were derived from an ATLAS9 stellar atmosphere model (Kurucz 1994) with $T_{\text {eff }}=12000 \mathrm{~K}$ and $\log \left[\mathrm{g} /\left(\mathrm{cm} \mathrm{s}^{-2}\right)\right]=2.00$. The data are summarized in Table 1.

We determined the total extinction (i.e., interstellar and circumstellar extinction combined) for each combination of observed photometric bands (excluding $V-R_{\mathrm{C}}$ ) such that the dereddened color would match the intrinsic color. We then averaged all the measurements of $A_{V}$ and determined the standard deviation. We used the extinction law from Cardelli et al. (1989) and determined $R_{V}$ by minimizing the standard deviation. The resulting values are: $A_{V}=7.5 \pm 0.4 \mathrm{mag}$, with $R_{V}=4.2$. Note that the uncertainty quoted here is the uncertainty in the average value, not the spread of the individual measurements. The galactic extinction estimate at the position of IRAS 16594-4656 is $A_{V}=6.6 \mathrm{mag}$ according to Schlegel et al. (1998), who assume an $R_{V}=3.1$ extinction curve. This value is in good agreement with our determination. The extinction value is considerably higher than the estimate $A_{V} \approx 3.2$ mag given by Hrivnak et al. (1999). The latter estimate pertains to the interstellar extinction component alone at an assumed distance of more than $1.3 \mathrm{kpc}$, and is based on the galactic extinction study by Neckel \& Klare (1980).

Visual inspection of the Digitized Sky Survey image taken in the direction of IRAS 16594-4656 reveals that the extinction is very patchy in the neighborhood of this object. Hence the use of estimates from galactic extinction studies should be considered very uncertain. On the other hand, some of the difference between the two extinction values could be due to significant circumstellar extinction, which would be supported by the large infrared excess of this object. However it is unclear how much of the total extinction is of circumstellar origin.

With this value for the extinction and the flux calibration from the Kurucz model, we can determine that the distance to IRAS $16594-4656$ is $(2.2 \pm 0.4) L_{4}^{1 / 2} \mathrm{kpc}$, where $L_{4}$ is the luminosity of IRAS $16594-4656$ in units of $10^{4} L_{\odot}$. This value is in good agreement with the distance of $2.5 L_{4}^{1 / 2} \mathrm{kpc}$ derived by Su et al. (2001), based on the dereddened bolometric flux of IRAS 16594-4656. The latter value may have been somewhat overestimated due to stellar radiation escaping through the lobes. The distance is also somewhat sensitive to uncertainties in the interstellar extinction estimate, although this effect is mild since most of the bolometric flux is emitted in the mid-infrared.

\section{Infrared lines}

\subsection{Atomic hydrogen}

The Br $\gamma$ line is in emission, but doesn't show a P-Cygni profile as we observed in the $\mathrm{H} \alpha$ line (Van de Steene et al. 2000b). The $\operatorname{Br} \gamma$ flux detected in the SOFI spectrum is approximately $40 \%$ lower than measured from the higher resolution IRSPEC spectra obtained in 1995 by Van de Steene et al. (2000a) and in 1994 by García-Hernández et al. (2002). For the IRSPEC observations the slit had a width of 4 .' 5 and was oriented $\mathrm{N}-\mathrm{S}$ and $\mathrm{E}-\mathrm{W}$, respectively, while in our SOFI observations the slit had a width of only $1^{\prime \prime}$ and was oriented E-W. Based on the continuum flux, both calibrations agree. This could indicate that we missed some line emission due to the narrow slit width in the SOFI observations. However, this is not a very plausible explanation. The $\mathrm{Br} \gamma$ emission is formed in the post-AGB wind, very close to the central star. The emission region is much smaller than $1^{\prime \prime}$ in diameter, so if we missed $\operatorname{Br} \gamma$ emission, we would also have missed continuum emission from the central star. Reflection also seems a very implausible explanation, since it is not expected that dust scattering in the infrared would be efficient enough to cause a $40 \%$ discrepancy in the observed fluxes. This leaves as the only alternative explanation that the strength of the Br $\gamma$ emission became less between 1994/5 and 1998. We have seen spectral variability in other, similar objects in our sample, so this possibility cannot be ruled out. Further observations will be needed to settle this point.

The $\operatorname{Pa} \beta$ line has been observed for the first time in this spectrum, and is shown in Fig. 3. 

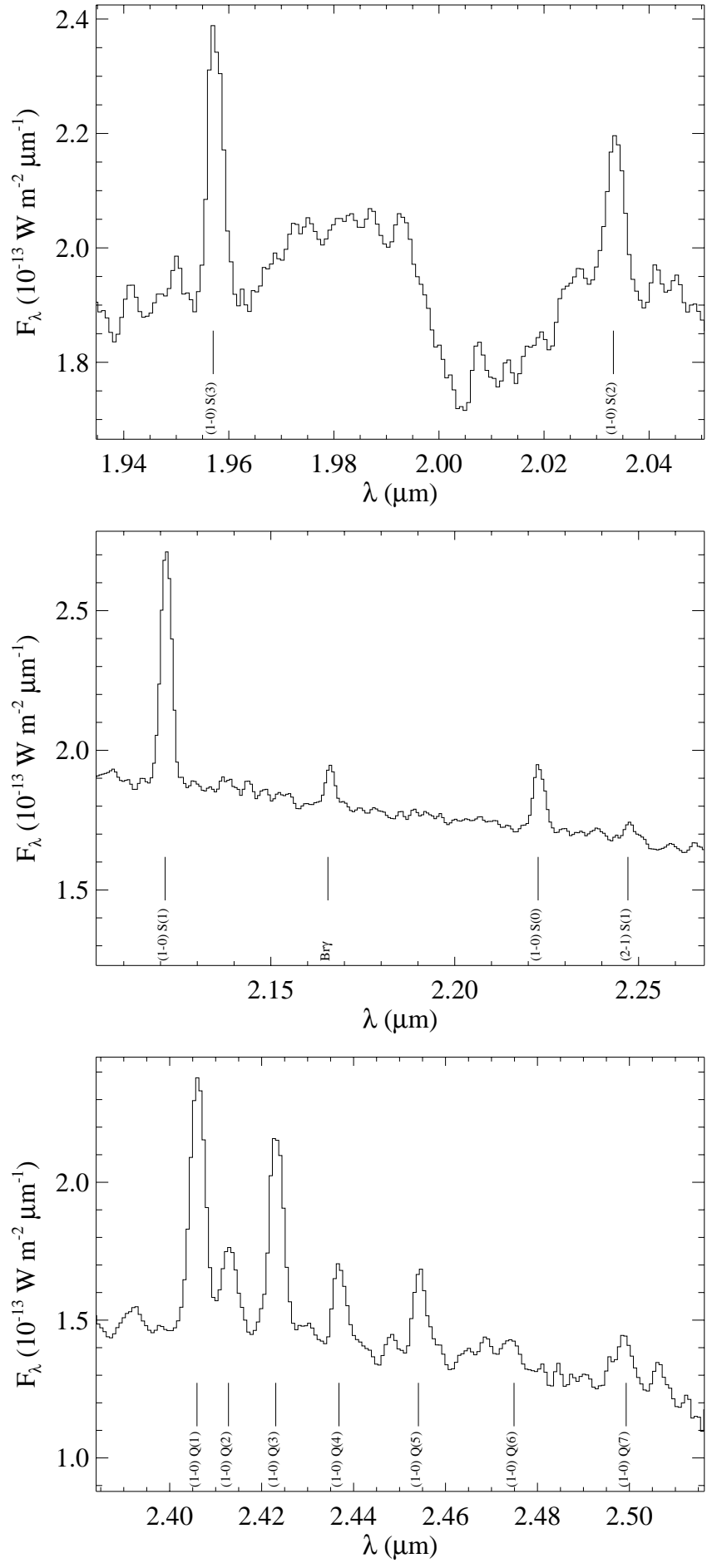

Fig. 2. The $\mathrm{H}_{2}$ spectrum of IRAS 16594-4656.

\subsection{Molecular hydrogen}

The observed $\mathrm{H}_{2}$ lines are shown in Fig. 2. The strongest $\mathrm{H}_{2}$ emission is seen from $(1,0) \mathrm{Q}(1),(1,0) \mathrm{S}(1)$, and $(1,0) \mathrm{Q}(3)$. The weakest line is $(2,1) \mathrm{S}(1)$ and no other lines with $v=2$ or higher are detected.

Compared with the IRSPEC value given by García-Hernández et al. (2002), we observe $~ 50 \%$ less flux for the $\mathrm{H}_{2}(1,0) \mathrm{S}(1)$ line. The flux of the faint $\mathrm{H}_{2}(2,1) \mathrm{S}(1)$ line however agrees for both observations (our detection has a
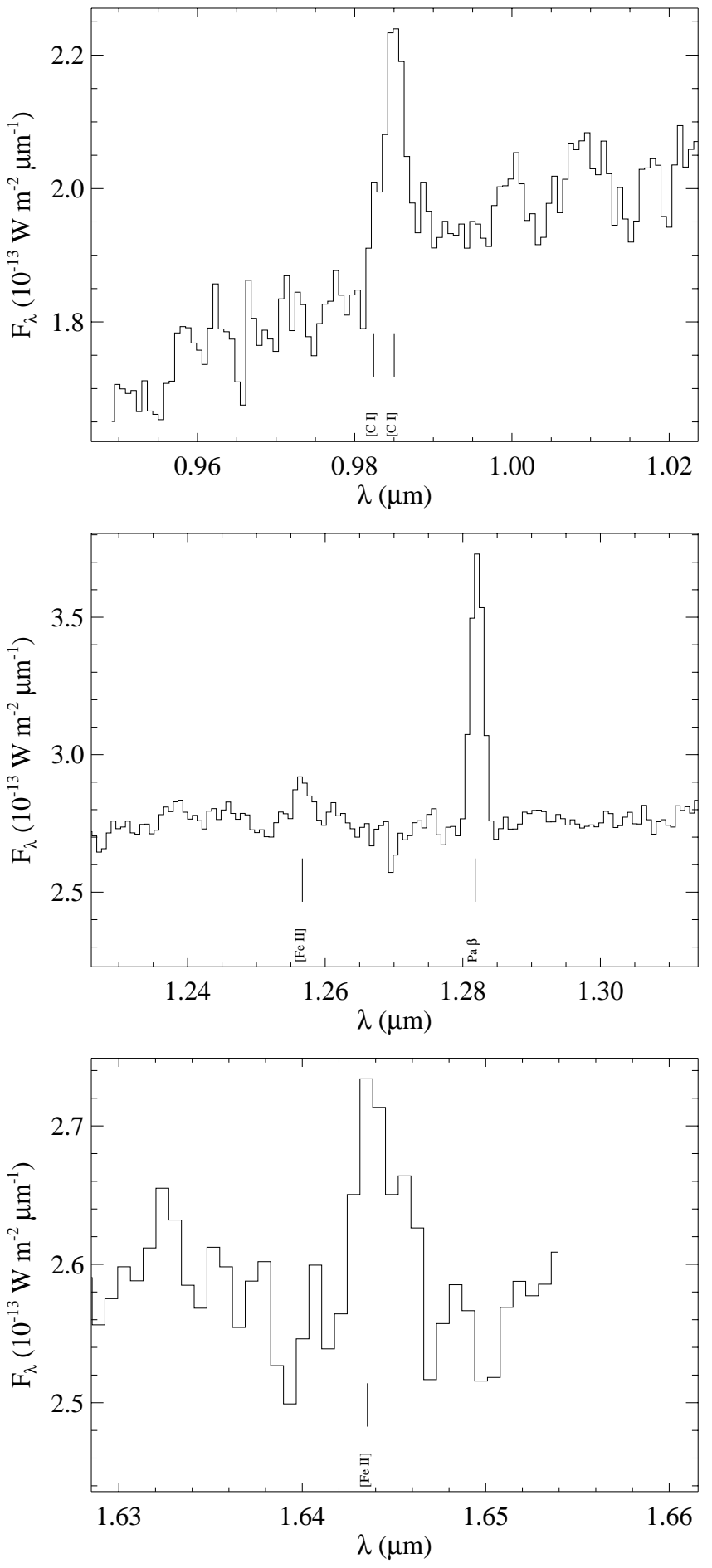

Fig. 3. The other emission lines of IRAS 16594-4656.

large error margin though). This discrepancy could either be due to flux missed outside the SOFI slit, or to variability in the post-AGB wind. See also the discussion in the previous subsection and Sect. 6.

\subsection{Carbon}

The [C I] 9824 \& $9850 \AA$ lines are metastable lines often observed in shocks (Hollenbach \& McKee 1989). Neutral carbon 
is readily ionized, so the existence of $[\mathrm{CI}]$ requires a region sheltered from ionizing radiation, or indicates the lack of it.

Both [C I] $9824 \AA$ and $9850 \AA$ come from the same upper level and have a fixed intensity ratio of approximately $1: 3$. The fact that [C I] $9824 \AA$ is not clearly detected in our spectrum is most likely due to telluric absorption.

\subsection{Iron}

[Fe II] lines are known to be good tracers of astrophysical shocks in supernovae, Herbig-Haro objects, PNe, etc. [Fe II] lines are very sensitive to the high densities and temperatures in the shocked gas. The excitation temperature of the upper term of the IR multiplets of [Fe II], which fall in atmospheric windows at 1.2 and $1.6 \mu \mathrm{m}$, is $2-3$ times lower than of optical lines and the critical densities of these [Fe II] lines are $\sim 10$ times higher.

The [Fe II] $a^{4} \mathrm{~F}-a^{4} \mathrm{D} 1.644 \mu \mathrm{m}$ over $\mathrm{Br} \gamma$ intensity ratio is often used as an indicator of shock excitation. The ratio expected for shock-excited gas is much larger than 1, but the [Fe II] $1.644 \mu \mathrm{m} / \mathrm{Br} \gamma$ ratio expected for radiatively excited gas is only approximately 0.06 (Graham et al. 1987). The ratio which we obtain for the dereddened line ratios is 0.9 , which is much larger than the value typically found in H II regions.

\section{Excitation of molecular hydrogen}

The excitation of molecular hydrogen has been the subject of several theoretical investigations which are reviewed by Sternberg (1990) and Burton (1992). Two principal sources of $\mathrm{H}_{2}$ emission are (1) photodissociation regions (PDRs), where the molecules are vibrationally excited by far-ultraviolet (FUV) pumping or collisionally excited in gas heated by FUV radiation; and (2) shocked regions, in which the hydrogen molecules are collisionally excited in hot gas behind the shock front. In post-AGB stars both forms of excitation may occur. Observations of the rich $\mathrm{H}_{2}$ ro-vibrational spectrum yield line ratios that are valuable probes of the physical conditions within the emitting source. These allow us to determine the excitation mechanism.

García-Hernández et al. (2002) concluded that fluorescent $\mathrm{H}_{2}$ emission becomes active when the central star reaches a temperature that corresponds to spectral type A. Shock excited $\mathrm{H}_{2}$ emission was only detected in objects with a marked bipolar morphology, sometimes at an early stage during the postAGB phase. Sometimes this emission was localized in the waist and/or in specific regions of the bipolar lobes, where a physical interaction exists between fast- and slow-moving material. In this section we investigate the excitation mechanism of $\mathrm{H}_{2}$ in IRAS 16594-4656.

\subsection{Rotational and vibrational temperatures of $\mathrm{H}_{2}$}

Because $\mathrm{H}_{2}$ is formed from two identical particles, only quadrupole transitions are allowed, and transition probabilities are very low. As a consequence, the radiative lifetimes of the levels are long and all levels will be populated thermally within a given vibrational state. If the relative strengths for a

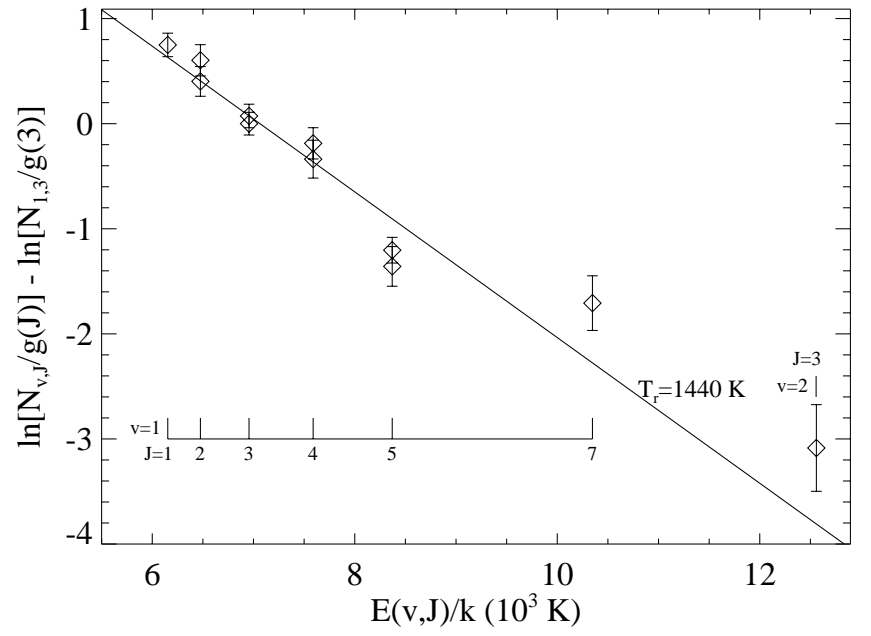

Fig. 4. The column densities for the various observed ro-vibrational levels of $\mathrm{H}_{2}$. The best-fit line based on a rotational temperature of $T_{\mathrm{r}}=1440 \mathrm{~K}$ is shown as well.

few of the transitions can be measured, then this information can be used to derive the rotation temperature for that vibrational state. If collisions alone excite molecular hydrogen, then populations in different vibrational states will also be in thermal equilibrium and all levels can be described by the same temperature. However, if the excitation mechanism includes a fluorescent component, higher vibrational states will be overpopulated, thus indicating a vibrational temperature higher than the rotational value. Hence, thermal excitation will give similar rotational and vibrational temperatures, while fluorescence is characterized by a high vibrational temperature and a low rotational temperature (Rudy et al. 2001).

Figure 4 provides a graphical determination of the temperature and excitation mechanism of the molecular hydrogen in IRAS $16594-4656$ in the standard manner (see, e.g., Martini et al. 1997). The $y$-axis shows the log of the relative column density of molecular hydrogen in a given state, $N(v, J)$, divided by the statistical weight, $g(J)$. These were derived by converting the reddening corrected line fluxes $F_{\text {cor }}$ into column densities for the upper levels using the formula:

$N(v, J)=\frac{F_{\mathrm{cor}} \lambda}{\Omega h c A_{\mathrm{ki}}}$.

Here all symbols have their usual meaning and $\Omega$ is the solid angle covered by the observations. The transition probabilities $A_{\mathrm{ki}}$ were taken from Martin et al. (1996). Since $\Omega$ is unknown for IRAS 16594-4656, we present the data as ratios to $N(1,3) / g(3)$. The $x$-axis gives the temperature of the upper level of a given transition.

Comparison of the population of upper levels with different rotational quantum numbers ( $J$-values), but identical vibrational quantum numbers ( $v$-values), provides an estimate for the rotational temperature. The temperature can be found directly from the slope of a straight line fitted to the data points. We have made a least-squares fit using the formula

$\ln y-\ln y_{0}=a+b x$

with $x=E(v, J) / k, y=N(v, J) / g(J)$ and $y_{0}=N(1,3) / g(3)$. The rotational temperature is simply given by $T_{\mathrm{r}}=-1 / b$. 
Table 2. The emission lines detected in the spectrum of IRAS 16594-4656. The observed flux values $F_{\text {obs }}$ in the 5th column are not corrected for extinction, but the $F_{\text {cor }}$ values in the last column are corrected for extinction.

\begin{tabular}{lllllll}
\hline \hline Line & $\begin{array}{l}\lambda_{\text {lab }} \\
\mu \mathrm{m}\end{array}$ & $\begin{array}{l}\lambda_{\text {obs }} \\
\mu \mathrm{m}\end{array}$ & $\begin{array}{l}\text { Continuum } \\
\mathrm{erg} \mathrm{cm}^{-2} \mathrm{~s}^{-1} \AA^{-1}\end{array}$ & $\begin{array}{l}F_{\text {obs }} \\
\mathrm{erg} \mathrm{cm}^{-2} \mathrm{~s}^{-1}\end{array}$ & $\begin{array}{l}F W H M \\
\mu \mathrm{m}\end{array}$ & $\begin{array}{l}F_{\text {cor }} \\
\mathrm{erg} \mathrm{cm}^{-2} \mathrm{~s}^{-1}\end{array}$ \\
\hline$(1,0) \mathrm{S}(3)$ & 1.9570 & 1.9575 & $1.91(-14)^{a}$ & $1.66(-13)$ & $3.13(-3)$ & $4.88(-13)$ \\
$(1,0) \mathrm{S}(2)$ & 2.0332 & 2.0336 & $1.93(-14)$ & $1.07(-13)$ & $3.69(-3)$ & $2.88(-13)$ \\
$(1,0) \mathrm{S}(1)$ & 2.1213 & 2.1216 & $1.88(-14)$ & $3.17(-13)$ & $3.36(-3)$ & $7.87(-13)$ \\
$(1,0) \mathrm{S}(0)$ & 2.2227 & 2.2229 & $1.71(-14)$ & $9.15(-14)$ & $3.61(-3)$ & $1.95(-13)$ \\
$(2,1) \mathrm{S}(1)$ & 2.2471 & 2.2478 & $1.67(-14)$ & $2.62(-14):$ & $4.01(-3):$ & $4.87(-14):$ \\
$(1,0) \mathrm{Q}(1)$ & 2.4059 & 2.4061 & $1.47(-14)$ & $3.71(-13)$ & $3.65(-3)$ & $7.78(-13)$ \\
$(1,0) \mathrm{Q}(2)$ & 2.4128 & 2.4130 & $1.43(-14)$ & $1.32(-13)$ & $3.88(-3)$ & $2.63(-13)$ \\
$(1,0) \mathrm{Q}(3)$ & 2.4231 & 2.4232 & $1.44(-14)$ & $2.91(-13)$ & $3.54(-3)$ & $5.94(-13)$ \\
$(1,0) \mathrm{Q}(4)$ & 2.4368 & 2.4370 & $1.42(-14)$ & $9.66(-14)$ & $3.04(-3)$ & $1.86(-13)$ \\
$(1,0) \mathrm{Q}(5)$ & 2.4541 & 2.4544 & $1.39(-14)$ & $9.91(-14)$ & $3.04(-3)$ & $2.02(-13)$ \\
$(1,0) \mathrm{Q}(7)$ & 2.4993 & 2.4989 & $1.22(-14)$ & $8.53(-14):$ & $4.25(-3):$ & $1.75(-13):$ \\
\hline$[\mathrm{C} \mathrm{I}]$ & $0.9844^{b}$ & 0.9849 & $1.88(-14)$ & $1.16(-13)$ & $2.71(-3):$ & $2.98(-12)$ \\
{$[\mathrm{Fe} \mathrm{II}]$} & 1.2567 & 1.2567 & $2.76(-14)$ & $3.88(-14):$ & $2.12(-3)$ & $3.23(-13):$ \\
$\mathrm{Pa} \beta$ & 1.2818 & 1.2821 & $2.73(-14)$ & $2.34(-13)$ & $2.09(-3)$ & $1.88(-12)$ \\
{$[\mathrm{Fe}$ II $]$} & 1.6435 & 1.6437 & $2.55(-14)$ & $3.66(-14)$ & $2.17(-3)$ & $8.70(-14)$ \\
$\mathrm{Br} \gamma$ & 2.1655 & 2.1662 & $1.80(-14)$ & $4.52(-14)$ & $2.91(-3)$ & $9.42(-14)$ \\
\hline
\end{tabular}

${ }^{a}$ Entries such as $(-14)$ stand for $\times 10^{-14}$.

${ }^{b}$ This line is a blend of the [C I] $0.9824 \& 0.9850 \mu \mathrm{m}$ lines.

The results are: $a=4.90 \pm 0.30, b=(-6.93 \pm 0.41) \times 10^{-4}$. Hence, the rotational temperature $T_{\mathrm{r}}=1440 \pm 80 \mathrm{~K}$.

The vibrational temperature is measured from the slope of a line passing through data points with different vibrational quantum numbers but the same rotational quantum number in the $\ln [N(v, J) / g(J)], T$-plane. We determined $T_{\text {vib }}=1820 \pm 240 \mathrm{~K}$.

The fact that the two values differ only by $1.6 \sigma$ is consistent with the assumption that fluorescence doesn't contribute strongly to the excitation and that in IRAS 16594-4656 the $\mathrm{H}_{2}$ excitation is mainly collisional.

\subsection{The ortho-to-para ratio}

The ortho-to-para ratio of molecular hydrogen is the ratio of the total column density of ortho- $\mathrm{H}_{2}$ (all odd $J$ states) to para- $\mathrm{H}_{2}$ (all even $J$ states).

If the ro-vibrational states are predominantly populated by collisional processes, the ortho-to-para ratio will be very close to 3. On the other hand, if FUV fluorescence plays an important role in populating the levels, the ortho-to-para ratio will be significantly smaller than 3 (Martini et al. 1997). Hence the orthoto-para ratio can be used as a diagnostic to determine whether FUV fluorescence plays a role in exciting the ro-vibrational states.

To calculate the ortho-to-para ratio from our observed line fluxes, we used Eq. (5) from Hoban et al. (1991) with the transition probabilities and level energies taken from Martin et al. (1996). Since we observed many more lines than they did, we chose to calculate the ortho-to-para ratio from each combination of ortho and para states that was observed in our spectrum. We also assigned an uncertainty to each ratio, based on the estimated uncertainties in the line fluxes and calibration. We then calculated the weighted average of all ratios, resulting in an ortho-to-para ratio of $2.77 \pm 0.19$. Note that the uncertainty quoted here is the uncertainty in the average value, not the spread of the individual measurements. This value is in good agreement with the expected ratio of 3 for collisionally excited molecular hydrogen.

\subsection{The $(1,0) S(1) /(2,1) S(1)$ ratio}

A diagnostic that has been commonly used to discriminate between shocks and FUV-pumped fluorescence, is the $(1,0) \mathrm{S}(1) /(2,1) \mathrm{S}(1)$ ratio. In shocks this ratio is typically $\sim 10$ (e.g., Shull \& Hollenbach 1978, and references therein) while for pure radiative fluorescence it is 1.8 (Black \& Dalgarno 1976). This ratio is essentially based on the same physics as the comparison between the rotational and vibrational temperatures, but is a useful diagnostic when no other $\mathrm{H}_{2}$ lines have been observed. The dereddened value we obtain for this ratio is 16.2 , thus indicating shock excitation.

\subsection{Thermal excitation}

Based on the evidence presented in the previous subsections, we can state that the $\mathrm{H}_{2}$ emission is collisionally excited by other atoms or molecules in the gas. These colliding particles could obtain their energy either from absorbing stellar UV photons with energies less than $11.2 \mathrm{eV}$ (more energetic photons cannot be present, they would dissociate $\mathrm{H}_{2}$ ), or from 
shocks. In order to investigate this point further, we examined the $12000 \mathrm{~K}$ post-AGB star model from run 4 with graphite dust and no dust formation in the post-AGB wind (van Hoof et al. 1997). This model should give a fair approximation for the physical conditions in IRAS 16594-4656. Using the distance estimate from Sect. 3 and the known angular dimensions of IRAS 16594-4656, we noticed that the radial sizes in this model were approximately a factor of two too large. Hence we reduced all radii by a factor of two and increased all densities by a factor of four. We then recalculated the model using Cloudy 96 beta 4 (Ferland 2002). This showed that in regions where molecular hydrogen is abundant, the electron temperature is $210 \mathrm{~K}$ or lower. This value is significantly lower than the rotational temperature of $1440 \mathrm{~K}$ which we derived above. It is therefore very unlikely that $\mathrm{H}_{2}$ is thermally excited by UV heated gas.

\section{Discussion}

Based on the evidence presented in the previous section, we can conclude that the $\mathrm{H}_{2}$ emission is collisionally excited by shocks in IRAS 16594-4656.

Molecular shocks generally can be described as " $C$-shocks" or as " $J$-shocks", depending on their shock velocity $v_{\mathrm{s}}$, the ambient ionization fraction and the strength of the component of the ambient magnetic field perpendicular to $v_{\mathrm{s}}$ (Draine 1980). A $J$-shock is a shock where the heat deposition length is short compared to the cooling length. $J$-shocks are sufficiently powerful to dissociate molecules. $C$-shocks occur at relatively low velocities and moderate to low ionizations and depend upon the presence of a magnetic field. In $C$-shocks, gas is accelerated and heated by collisions between charged particles and neutral particles. $C$-shocks cool mainly through molecular material and grains. In $C$-shocks the radiation is emitted as the gas is being heated in the shock front; in $J$-shocks it is emitted after the impulsive heating event, downstream behind the shock front.

The $\mathrm{H}_{2}$ emission in IRAS 16594-4656 appears strong: much stronger than $\mathrm{Br} \gamma$ for instance. Molecular lines produced by collisional excitation of $\mathrm{H}_{2}$ will be strongest if the collisions are not energetic enough to dissociate $\mathrm{H}_{2}$ and lower its abundance (Hollenbach \& McKee 1989). Such conditions exist in $C$-shocks because the gas is heated gradually and remains molecular. Hence large columns of material with temperatures between $1000 \mathrm{~K}$ and $3000 \mathrm{~K}$ can be produced which generate copious amounts of $\mathrm{H}_{2}$ emission. Therefore the strong $\mathrm{H}_{2}$ emission in IRAS 16594-4656 argues in favor of a $C$-shock.

We compared our line ratios of the $\mathrm{H}_{2}$ emission with the intensities of ro-vibrational transitions of $\mathrm{H}_{2}$ from $C$-shock models by Le Bourlot et al. (2002). Our values are consistent with a $20-30 \mathrm{~km} \mathrm{~s}^{-1} C$-shock propagating in $10^{3} \mathrm{~cm}^{-3}$ material. They are not consistent with any of their other models where the $C$-shocks have either a higher velocity or impinge on higher density gas.

However, $C$-type shocks depend upon the presence of a magnetic field. There is no direct evidence for the presence of a magnetic field in IRAS 16594-4656. Su et al. (2003) observed $10 \%$ polarization close to the central star, which makes it likely that the EDE harbors one. Direct detection of magnetic fields is very difficult, and has only been attempted a handful of times. Nevertheless, a detection has been achieved in four postAGB stars and PNe. Miranda et al. (2001) detected a toroidal magnetic field in the EDE of the young PN K3-35. Greaves (2002) detected a toroidal magnetic field around NGC 7027 with an effective scale of $5000 \mathrm{AU}$ and correctly oriented to collimate the observed bipolar winds, and of a dimension similar to the base of the outflow. They also detected a magnetic field in CRL 2688. Bains et al. (2003) measured for the first time the magnetic field strength in a post-AGB star. They found $B=+4.6 \mathrm{mG}$ at $1612 \mathrm{MHz}$ and $B=+2.5 \mathrm{mG}$ at $1667 \mathrm{MH}$ in $\mathrm{OH} 17.7-2.0$.

The lack of significant ionization (no radio emission or lines typical of $\mathrm{H}$ II regions have been detected) is consistent with the prerequisites of a $C$-type shock.

However, metastable lines such as [OI] $6300 \AA$ and [CI] $9850 \AA$ have been detected. These are only produced in $J$-shocks. $C$-shocks, which have large molecular abundances, tend to rapidly convert any pre-existing atomic oxygen into molecular form (Hollenbach \& McKee 1989). The observation of ionic lines such as [Fe II] are also a strong indication of $J$-shocks, if one has eliminated the possibility of an H II region as the origin. Few ions exist in $C$-shocks, which only form when the shocked gas is largely neutral and molecular.

Another indication in favor of the presence of a $J$-shock is the wind speed of $126 \mathrm{~km} \mathrm{~s}^{-1}$ derived from the $\mathrm{H} \alpha$ P-Cygni profile (Van de Steene et al. 2000b). This velocity is too high for a $C$-shock, but consistent with velocities for a $J$-type shock (Hollenbach \& McKee 1989).

[C I] $9850 \AA$, [N I] $1.040 \mu \mathrm{m}$, and [Fe II] $1.257 \& 1.644 \mu \mathrm{m}$ are all predicted to be strong near-infrared emission lines in $J$-type shocks, while in the optical [N II] 6548 \& $6584 \AA$, and [S II] $6716 \& 6731 \AA$ would be strong. Comparing the detected near-infrared line fluxes to the model predictions presented in Fig. 8 of Hollenbach \& McKee (1989), we find that they are compatible with an $80 \mathrm{~km} \mathrm{~s}^{-1} \mathrm{~J}$-shock impinging upon $10^{3} \mathrm{~cm}^{-3}$ material, or alternatively a $70 \mathrm{~km} \mathrm{~s}^{-1}$ shock impinging upon $10^{4} \mathrm{~cm}^{-3}$ material. This would be consistent with a $126 \mathrm{~km} \mathrm{~s}^{-1}$ wind colliding against the side walls of an hourglass nebula at an angle of approximately $45^{\circ}$ w.r.t. the normal, assuming that the side walls are expanding at a rate of $16 \mathrm{~km} \mathrm{~s}^{-1}$ (Loup et al. 1990). This value for the impact angle is in fair agreement with the wide opening angle of the lobes seen in the image in Su et al. (2001).

In the optical (Reyniers, private communication) and infrared spectra no forbidden lines from ionized species other than [Fe II] were detected. A weak detection of [N II] 6548 \& $6584 \AA$ would be expected in the optical spectrum according to the $10^{3} \mathrm{~cm}^{-3}$ model of Hollenbach \& McKee (1989). For a given shock velocity, the ionized states will become relatively weaker for higher densities since the overall degree of ionization will shift downwards. Taken at face value this could point to a relatively high density (at least $10^{4} \mathrm{~cm}^{-3}$ ) at the edge of the lobes. However, according to the $10^{4} \mathrm{~cm}^{-3}$ model, a weak detection of [N I] $1.040 \mu \mathrm{m}$ would be expected, which was not observed in our infrared spectrum either. The critical density of this line at $T_{\mathrm{e}}=10^{4} \mathrm{~K}$ is approximately $10^{7} \mathrm{~cm}^{-3}$, using data from Zeippen (1982) and Dopita et al. (1976). 
This value is larger than for any of the other lines listed above, ruling out an extremely high density and collisional de-excitation of the line as the cause of the non-detection. Consequently the non-detections of [N I] $1.040 \mu \mathrm{m}$ and [N II] $6548 \& 6584 \AA$ seem to contradict each other and could be indicative that IRAS 16594-4656 has a low nitrogen abundance. Bipolar PNe with high $\mathrm{C} / \mathrm{O}$ and low $\mathrm{N} / \mathrm{O}$ ratios are known (e.g., J 900, Perinotto 1991), although bipolar PNe are usually associated with type I PN, which have high nitrogen abundances. One should keep in mind though that the Hollenbach \& McKee (1989) models use solar abundances. The carbon-rich chemistry in IRAS 16594-4656 could have an important effect on the models since it will alter the cooling significantly. Deeper observations are needed to settle this point.

From the previous discussion it is seems that slower $C$-type and faster $J$-type shocks both are present in IRAS 16594-4656. Because the ionization potential of neutral iron is $7.87 \mathrm{eV}$, and the dissociation energy of $\mathrm{H}_{2}$ is $4.48 \mathrm{eV}$ (Graham et al. 1987), in principle $\mathrm{H}_{2}$ and $\mathrm{Fe}^{+}$cannot coexist in the same region in substantial quantities. Hence, the $C$ - and $J$-shocks must occur in different regions in the nebula.

$C$-shocks would occur where the shock velocity is low, hence where the fast wind impinges almost tangentially upon the circumstellar material. The $\mathrm{H}_{2}$ emission would originate from this region. We postulate that the $C$-shocks occur where the fast wind is funneled through the EDE of IRAS 16594-4656. This is in agreement with Guerrero et al. (2000) who noted that, for PNe, in all cases where the emission observed in the $\mathrm{H}_{2}$ line is more than 5 times greater than the $\mathrm{Br} \gamma$ emission, the $\mathrm{H}_{2}$ emission is mainly concentrated in a bright ring, the "waist" of the bipolar nebula. For IRAS 16594-4656 the Br $\gamma$ line is much weaker than the $\mathrm{H}_{2}(1,0) \mathrm{S}(1)$ line. The ratio $\mathrm{H}_{2}(1,0) \mathrm{S}(1)$ to $\mathrm{Br} \gamma$ is 8.4 after correction for extinction. As mentioned above, the EDE is likely to harbor a magnetic field, which is a prerequisite for a $C$-shock.

However, molecules have been known to survive in dense condensations and cometary knots (e.g., Speck et al. 2002). Hence it cannot be excluded that some $\mathrm{H}_{2}$ emission is present in the lobes, excited by shocks caused by a molecular outflow impinging on the AGB envelope (e.g., the Egg Nebula, Cox et al. 2000). The large difference seen between the IRSPEC and $S O F I$ value of $\mathrm{H}_{2}(1,0) \mathrm{S}(1)$ may support this. However, no collimated outflow nor high density blobs have been observed in IRAS 16594-4656 yet.

$J$-shocks occur where the shock velocity is high. A first possibility is that the $[\mathrm{CI}]$ and [Fe II] line emission originates in a $J$-shock along the walls of the bipolar lobes, where the fast wind impinges with a high transverse velocity. This is in agreement with the observations of Hora \& Latter (1996) and Welch et al. (1999) who imaged the shock excited [Fe II] line emission in the young PN Hubble 12. The shape of this PN resembles a tilted hourglass. The [Fe II] emission is located along the side walls of the hourglass in this object and its morphology differs strikingly from that of the $\mathrm{H}_{2}$ emission, which is mostly dominated by UV excitation. The geometry of this PN is very similar to IRAS $16594-4656$, but Hubble 12 is a PN and hence at a much more evolved stadium.

A second possibility is that the [Fe II] emission originates much closer to the central star, in the post-AGB wind itself. Shock waves induced by stellar pulsations were proposed to explain the [Fe II] emission in Mira variables (Richter et al. 2003). Post-AGB stars are of course hotter than Miras, but they are usually variable and still pulsating. Fokin et al. (2001) argue that stellar pulsations are forming shocks in the atmosphere of the post-AGB star HD 56126. Miras are believed to have very high mass loss rates, but the mass loss rate in post-AGB stars may still be quite large (up to $10^{-5}-10^{-6} M_{\odot} \mathrm{yr}^{-1}$, Gauba et al. 2003), as may be indicated by the strong P-Cygni Balmer lines in the optical spectrum of IRAS 16594-4656. Moreover, postAGB stars have higher wind velocities, which lead to the same normalized [Fe II] peak fluxes at lower pre-shock densities.

A third possibility is that the $J$-shocks would occur where matter transferred from a binary companion hits an accretion disk. At this stage there is no corroborating evidence that either a binary companion or an accretion disk exists in IRAS 16594-4656.

High resolution images in $\mathrm{H}_{2}$ and in selected metastable lines will clarify which shock mechanism occurs where, while high spectral and spatial resolution infrared spectra will clarify the kinematics around the central star and in the bipolar lobes.

\section{Conclusions}

We examined the near-infrared spectrum of IRAS 16594-4656. It shows strong $\mathrm{H}_{2}$ emission lines and some typical metastable shock excited lines such as [Fe II] $1.257 \& 1.644 \mu \mathrm{m}$. We argue that the molecular hydrogen emission is mainly collisionally excited. Its strength indicates that the $\mathrm{H}_{2}$ emission originates in $C$-type shocks. However, the metastable lines, and especially the [Fe II] emission lines, indicate the presence of $J$-type shocks. These shocks don't usually coexist. Hence we postulate that the $\mathrm{H}_{2}$ emission originates mainly where the stellar wind is funneled through an EDE and therefore impinges almost tangentially upon the circumstellar material. The [Fe II] emission either occurs along the walls of the bipolar lobes where the transverse shock velocity would be higher, or could originate much closer to the central star in shocks in the post-AGB wind itself, or possibly in an accretion disk.

IRAS 16594-4656 is unique in the sense that it allows us to study shock excited lines which are not compromised by ionization or fluorescence. Further high resolution near-infrared spectra are currently being obtained in order to clarify the kinematics of this object and the shock mechanisms.

Acknowledgements. PvH thanks the Engineering and Physical Sciences Research Council of the UK for financial support. GVdS acknowledges support for this research carried out in the framework of project IUAP P5/36 financed by the Belgian Federal Office for Scientific, Technical and Cultural Affairs. This paper contains atomic line data obtained from the Atomic Line List v2.04, available at http://www.pa.uky.edu/ peter/atomic. We thank J. C. Weingartner for stimulating discussions. This research has made use of NASA's Astrophysics Data System. The photoionization code Cloudy was used, which is written by Gary Ferland at the University of Kentucky. 


\section{References}

Bains, I., Gledhill, T. M., Yates, J. A., \& Richards, A. M. S. 2003, MNRAS, 338, 287

Black, J. H., \& Dalgarno, A. 1976, ApJ, 203, 132

Burton, M. G. 1992, Aust. J. Phys., 45, 463

Cardelli, J. A., Clayton, G. C., \& Mathis, J. S. 1989, ApJ, 345, 245

Cox, P., Lucas, R., Huggins, P. J., et al. 2000, A\&A, 353, L25

Dopita, M. A., Mason, D. J., \& Robb, W. D. 1976, ApJ, 207, 102

Draine, B. T. 1980, ApJ, 241, 1021

Ferland, G. J. 2002, University of Kentucky, Physics Department Internal Rep.

Fokin, A. B., Lèbre, A., Le Coroller, H., \& Gillet, D. 2001, A\&A, 378, 546

García-Hernández, D. A., Manchado, A., García-Lario, P., et al. 2002, A\&A, 387, 955

García-Lario, P., Manchado, A., Ulla, A., \& Manteiga, M. 1999, ApJ, 513, 941

Gauba, G., Parthasarathy, M., Kumar, B., Yadav, R. K. S., \& Sagar, R. 2003, A\&A, 404, 305

Graham, J. R., Wright, G. S., \& Longmore, A. J. 1987, ApJ, 313, 847

Greaves, J. S. 2002, A\&A, 392, L1

Guerrero, M. A., Villaver, E., Manchado, A., García-Lario, P., \& Prada, F. 2000, ApJS, 127, 125

Hauschildt, P. H., Allard, F., Ferguson, J., \& Baron, E. 1999, ApJ, 512, 377

Hoban, S., Reuter, D. C., Mumma, M. J., \& Storrs, A. D. 1991, ApJ, 370,228

Hollenbach, D., \& McKee, C. F. 1989, ApJ, 342, 306

Hora, J. L., \& Latter, W. O. 1996, ApJ, 461, 288

Hora, J. L., Latter, W. B., \& Deutsch, L. K. 1999, ApJS, 124, 195

Hrivnak, B. J., Kwok, S., \& Su, K. Y. L. 1999, ApJ, 524, 849

Hrivnak, B. J., Kwok, S., \& Su, K. Y. L. 2001, AJ, 121, 2775

Kastner, J. H., Weintraub, D. A., Gatley, I., Merrill, K. M., \& Probst, R. G. 1996, ApJ, 462, 777

Kurucz, R. L. 1994, Solar abundance model atmospheres for 0, 1, 2, 4, and $8 \mathrm{~km} \mathrm{~s}^{-1}$, Kurucz CD-ROM No. 19, Smithsonian Astrophysical Observatory (Cambridge, Mass.)
Le Bourlot, J., Pineau des Forêts, G., Flower, D. R., \& Cabrit, S. 2002 MNRAS, 332, 985

Loup, C., Forveille, T., Nyman, L. Å., \& Omont, A. 1990, A\&A, 227, L29

Martin, P. G., Schwarz, D. H., \& Mandy, M. E. 1996, ApJ, 461, 265

Martini, P., Sellgren, K., \& Hora, J. L. 1997, ApJ, 484, 296

Miranda, L. F., Gómez, Y., Anglada, G., \& Torrelles, J. M. 2001, Nature, 414, 284

Neckel, Th., \& Klare, G. 1980, A\&AS, 42, 251

Perinotto, M. 1991, ApJS, 76, 687

Richter, He., Wood, P. R., Woitke, P., Bolick, U., \& Sedlmayr, E. 2003, A\&A, 400, 319

Rudy, R. J., Lynch, D. K., Mazuk, S., Puetter, R. C., \& Dearborn, D. S. P. 2001, AJ, 121, 362

Schlegel, D. J., Finkbeiner, D. P., \& Davis, M. 1998, ApJ, 500, 525

Shull, J. M., \& Hollenbach, D. J. 1978, ApJ, 220, 525

Speck, A. K., Meixner, M., Fong, D., et al. 2002, AJ, 123, 346

Sternberg, A. 1990, in Molecular Astrophysics, ed. T. W. Hartquist (Cambridge: Cambridge University Press), 384

Su, K. Y. L., Kwok, S., \& Hrivnak, B. J. 2000, Am. Astron. Soc. Meet. 197, \#131.03

Su, K. Y. L., Hrivnak, B. J., \& Kwok, S. 2001, AJ, 122, 1525

Su, K. Y. L., Hrivnak, B. J., Kwok, S., \& Sahai, R. 2003, AJ, in press [astro-ph/0304400]

Van de Steene, G. C., \& Pottasch, S. R. 1993, A\&A, 274, 895

Van de Steene, G. C., van Hoof, P. A. M., \& Wood, P. R. 2000a, A\&A, 362,984

Van de Steene, G. C., Wood, P. R., \& van Hoof, P. A. M. 2000b, in ASP Conf. Ser. 199, Asymmetrical Planetary Nebulae II: From Origins to Microstructures, ed. J. H. Kastner, N. Soker, \& S. Rappaport, 191

van Hoof, P. A. M., Oudmaijer, R. D., \& Waters, L. B. F. M. 1997, MNRAS, 289, 371

Weintraub, D. A., Huard, T., Kastner, J. H., \& Gatley, I. 1998, ApJ, 509, 728

Welch, C. A., Frank, A., Pipher, J. L., Forrest, W. J., \& Woodward, C. E. 1999, ApJ, 522, L69

Zeippen, C. J. 1982, MNRAS, 198, 111 